\title{
Optically Tunable Photonic Stop Bands in Homogeneous Absorbing Media
}

\author{
M. Artoni ${ }^{1,2}$ and G. C. La Rocca ${ }^{3}$ \\ ${ }^{1}$ Department of Chemistry and Physics of Materials, University of Brescia, Italy \\ ${ }^{2}$ European Laboratory for Non-Linear Spectroscopy, Sesto Fiorentino, Italy \\ ${ }^{3}$ Scuola Normale Superiore and CNISM, Pisa, Italy \\ (Received 11 August 2005; published 24 February 2006)
}

\begin{abstract}
Resonantly absorbing media supporting electromagnetically induced transparency may give rise to specific periodic patterns where a light probe is found to experience a fully developed photonic band gap yet with negligible absorption everywhere. In ultracold atomic samples the gap is found to arise from spatial regions where Autler-Townes splitting and electromagnetically induced transparency alternate with one another and detailed calculations show that accurate and efficient coherent optical control of the gap can be accomplished. The remarkable experimental simplicity of the control scheme would ease quantum nonlinear optics applications.
\end{abstract}

DOI: 10.1103/PhysRevLett.96.073905

The ability to mold the flow of light in complex photonic structures, of which photonic crystals [1] are the most familiar ones, is a fundamental issue of scientific and practical importance. Photonic crystals are naturally or artificially structured inhomogeneous materials in which the refractive index varies periodically over a length scale comparable to optical wavelengths. Because their periodic structure leads to Bragg scattering of an incident probe light beam, lightwave propagation in such crystals becomes best described in terms of a photonic band structure $[2,3]$ with band gaps where light does not propagate, akin to the electronic band gaps in crystalline solids.

The specific spatial dependence of the optical response in typical photonic crystals and the corresponding photonic band structure are determined once and for all by the way the material periodic structure is grown [1]. The need to design and make photonic crystals with predetermined energy bands could, however, be avoided if one could change their band structure by independent means. Some schemes to tune the band gap structure, e.g., have so far relied on slow electro- and thermo-optics effects of infiltrated liquid crystals [4] or on fast resonant and nonresonant optical nonlinearities in semiconductors [5]. In both instances, modifications do not affect the Brillouin zone while the gross features of the photonic bands remain set by the preassembled periodic structure.

We here follow a different approach and show that for a certain class of materials a periodic modulation of the medium optical response can actually be created through externally induced optical nonlinearities. Our specific scheme requires highly effective quantum coherence and interference effects to occur as those commonly observed in multilevel configurations leading to electromagnetically induced transparency [6]. In its simplest form such a transparency is exhibited in a three-level "lambda" configuration by a probe in the presence of a plane-wave pump beam [7]. The possibility of using instead a standing-wave pump beam anticipated in Ref. [8] is here exploited to generate photonic periodic structures where regions of weak and
PACS numbers: 42.70.Qs, 03.75.-b, 42.30.Rx, 42.50.Gy

strong normal dispersion periodically alternate with one another. The Brillouin zone structure and the band properties are in this case fully determined by the external pump control parameters, namely, its polarization, intensity, and detuning.

Although optically induced photonic lattices have been examined in a variety of situations including applied microscopy [9], soliton dynamics [10], quantum transport effects [11,12], and trapping potential for Bose-Einstein condensates [13], just to mention a few, one-dimensional lattices that involve periodically modulated electromagnetically induced transparency have been only recently explored [14]. These are sought after as a promising avenue, e.g., toward deterministic photon-photon entanglement [15] or the enhancement of nonlinear interactions between weak light pulses [16]. These lattices may also be used to reversibly convert a light pulse into a stationary excitation inside a dilute volume of atoms [17] where, however, resonant probe absorption still takes place at the pump nodes preventing [18] the full development of a photonic band gap. In spite of absorption, we anticipate that our specific standing-wave pump configuration leads to a full band gap, completely overcoming the detrimental effects of absorption. Because the underlying physical process is quite general the scheme may well be used for both atomic and solid media.

The idea is presented in the following for a homogeneous sample of cold atoms having a ground $(g)$, excited $(e)$, and metastable level $(m)$ in a standard three-level lambda configuration [7]. The transitions $e-g$ and $e-m$ are electric dipole allowed where $g$ is essentially the only populated level. Our results will be appropriate to the $D_{2}$ line of cold rubidium atoms in which case $g$ and $m$ are long-lived hyperfine sublevels of the electronic ground state $S_{1 / 2}$, while $e$ corresponds to the excited $P_{3 / 2}$ state. A weak probe beam of frequency $\omega$ and propagating in the $x$ direction is nearly resonant with the $e-g$ transition, while a strong pump or coupling beam of frequency $\omega_{c}$ and Rabi frequency $\Omega_{o}$ drives the $e-m$ transition. Unlike in typical 
lambda configurations leading to electromagnetically induced transparency $[6,7]$ where a $c w$ pump beam is used, the pump is here retro-reflected upon impinging on a mirror of reflectivity $R_{m}$ forming a standing-wave pattern within the sample [19]. The resulting Rabi frequency varies periodically along $x$,

$$
\frac{\Omega_{c}^{2}(x)}{\Omega_{o}^{2}}=\left(1+\sqrt{R_{m}}\right)^{2} \cos ^{2}\left(\frac{\omega_{c}}{c} x\right)+\left(1-\sqrt{R_{m}}\right)^{2} \sin ^{2}\left(\frac{\omega_{c}}{c} x\right),
$$

with a spatial periodicity $a$ which is half the pump beam wavelength $\lambda_{c}$. For a perfect $\left(R_{m}=1\right)$ standing wave $\Omega_{c}^{2}(x)$ varies periodically between 0 and $4 \Omega_{o}^{2}$ and vanishes at the nodal positions where the medium is absorbing. By slightly reducing the mirror reflectivity the pump intensity can, however, be made to vanish nowhere, as shown in the inset of the lower panel of Fig. 1, and the nodes will be replaced by quasinodes. In terms of the parameter $\eta=\left(1-\sqrt{R_{m}}\right) /\left(1+\sqrt{R_{m}}\right)$, where $0 \leq \eta \ll 1$, the minimum value of $\Omega_{c}^{2}(x)$ at a quasinode is approximately $4 \eta^{2} \Omega_{o}^{2}$. Because the probe is weak, levels $m$ and $e$ remain empty regardless of the pump intensity. As a consequence, the standing-wave intensity profile and its space periodicity do not alter the atoms space distribution, which then remains homogeneous within the sample. Conversely, the strong pump intensity pattern modifies in a periodic fashion the probe absorption and dispersion as one moves along $x$ from quasinodes to antinodes. In particular, at the quasinodes nearly complete suppression of the probe absorption with a concomitant steep dispersion occurs and within a very narrow window centered around the $e-g$ transition frequency $\omega_{o}$.

The refractive index experienced by the probe can be described by a dressed dielectric function [6] which, for a resonant pump, can be written as

$$
\epsilon(\omega, x)=1-3 \pi \mathcal{N} \frac{\gamma\left(\delta+i \gamma_{m}\right)}{(\delta+i \gamma)\left(\delta+i \gamma_{m}\right)-\Omega_{c}^{2}(x)} .
$$

Here $\delta=\omega-\omega_{o}$ is the probe detuning, $\mathcal{N}=\lambda_{0}^{3} n$ is the scaled average atomic density and $t_{o}=780.792 / 2 \pi \mathrm{nm}$ the reduced probe resonant wavelength. The excited and metastable levels' overall dephasings for ultracold ${ }^{87} \mathrm{Rb}$ atoms are $\gamma / 2 \pi \simeq 6 \mathrm{MHz}$ and $\gamma_{m} / 2 \pi \simeq 1 \mathrm{kHz}$, respectively. Owing to the induced periodic modulation (2) a probe with frequency $\omega \simeq \omega_{o}$ propagates as in a multilayer periodic structure with periodicity $a=\lambda_{c} / 2$ where $\lambda_{c} \approx$ $\lambda_{o}$. A photonic stop band is expected to occur at Brillouin zone boundary $\pi / a$.

To see this a $2 \times 2$ unimodular transfer matrix $M(\omega)$ describing probe propagation through a single period of length $a$ is first calculated numerically [20]. Then, by requiring translational invariance, Bloch conditions [21] must be imposed on the photonic eigenstates, i.e.,
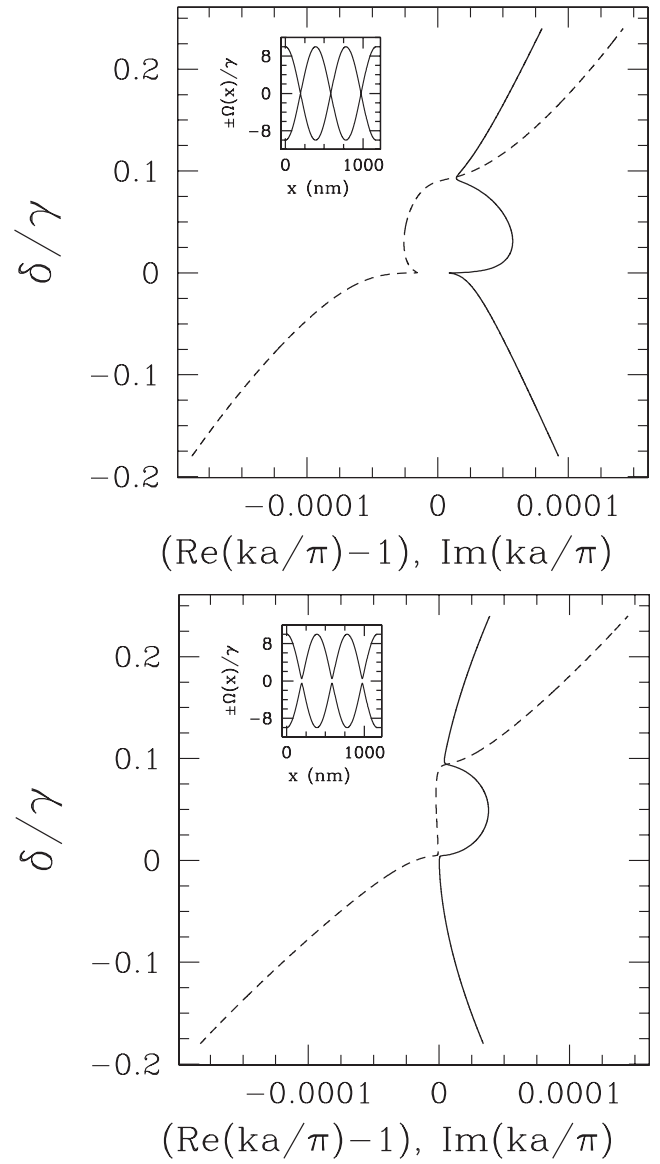

FIG. 1. Band gap structure for a probe in a homogeneous sample of ultracold ${ }^{87} \mathrm{Rb}\left(n=10^{12} \mathrm{~cm}^{-3}\right)$ in the presence of a periodic modulation induced by a perfect (upper panel $\eta=0$ ) and modified (lower panel $\eta=0.05$ ) standing-wave pattern with $\Omega_{o}=5 \gamma$. Both real (dashed line) and imaginary (solid line) parts of the probe Bloch wave vector are shown. The insets show the corresponding optically induced lattice intensity profiles.

$$
\left(\begin{array}{c}
E^{+}(x+a) \\
E^{-}(x+a)
\end{array}\right)=M(\omega)\left(\begin{array}{c}
E^{+}(x) \\
E^{-}(x)
\end{array}\right)=\left(\begin{array}{c}
e^{i k a} E^{+}(x) \\
e^{i k a} E^{-}(x)
\end{array}\right)
$$

where $E^{ \pm}$are the electric field amplitudes for the forward and backward Bragg reflected probe while its Bloch wave vector $k=k^{\prime}+i k^{\prime \prime}$ in (3) is, in general, complex. The photonic band structure is then obtained from solutions of the equation $e^{2 i k a}-\operatorname{Tr}[M(\omega)] e^{i k a}+1=0$ with $\operatorname{det} M=1$; noting that if $k$ is a solution so is $-k$, one obtains

$$
k a= \pm \cos ^{-1}\left[\frac{\operatorname{Tr} M(\omega)}{2}\right]
$$

We compare in Fig. 1 the band structure around the lowest photonic gap at the $X$ symmetry point for a perfect standing wave $(\eta=0)$ and that for a modified one $(\eta=$ 0.05). In the latter case, dissipation is negligible and a forbidden gap opens up in the frequency range for which $k^{\prime}=\pi / a$ and $k^{\prime \prime} \neq 0$ while $k^{\prime \prime} \simeq 0$ in the band. In the 
former case, residual absorption at the pump nodal positions is instead appreciable and the gap structure becomes barely defined [18]. In this case $k^{\prime}$ deviates, in fact significantly, from $\pi / a$ while $k^{\prime \prime}$ becomes appreciable within the allowed bands and asymmetric around the edges of the gap due to the Bormann effect [22].

The photonic band structure discussed in Fig. 1 refers to Bloch modes for a probe in an infinite periodic stack, yet typical experiments $[1,17]$ focus on propagation through samples of finite length. If one expresses the transfer matrix $M_{(N)}$ of a sample of thickness $L=N a$ in terms of the single period transfer matrix $M$ in (3) as

$$
M_{(N)}=M^{N}=\frac{\sin N k a}{\sin k a} M-\frac{\sin (N-1) k a}{\sin k a} \mathbf{1},
$$

where 1 denotes the unit matrix and the frequency dependencies are for simplicity omitted, the sample reflection $r$ and transmission $t$ amplitudes can be given in terms of the single period matrix elements $M_{i j}$ as

$$
r=\frac{M_{(N) 12}}{M_{(N) 22}}=\frac{M_{12} \sin N k a}{M_{22} \sin N k a-\sin (N-1) k a}
$$

and

$$
t=\frac{1}{M_{(N) 22}}=\frac{\sin k a}{M_{22} \sin N k a-\sin (N-1) k a} .
$$

These directly yield the probe reflection $\left(R=|r|^{2}\right)$ and transmission $\left(T=|t|^{2}\right)$ spectra both shown in Fig. 2 for a fairly thick sample. Unlike the case of a perfect $(\eta=0)$ standing wave [23] where absorption is significant in the spectral region of the gap, the use of a modified standing wave $(\eta=0.05)$ leads to a fully developed stop band with nearly [24] 100\% reflectivity. The narrow band gap is all contained within the transparency window at the quasinodes where the pump intensity is minimal [25]. These large reflectivities arise from efficient constructive interference of in-phase contributions from multiple reflections between adjacent atomic layers, a regime that is hard to reach as in resonant absorbing media Bragg scattering becomes strong only very close to resonance and where, however, absorption limits coherent multiple reflections.

We further show in Fig. 3 how to tune gap position and width by changing the lattice period and the pump Rabi frequency $\Omega_{o}$, while keeping $\eta$ fixed. By slightly misaligning the retro-reflected pump beam one can, in fact, easily change the spatial periodicity $a$ into $a / \cos (\chi / 2)$ where $\chi$ is the two counter-propagating beams' misalignment along $x$. Very small values of $\chi$ are enough to move the gap across resonance and make it just twice as wide as the one [26] shown in Fig. 2. The gap may instead be narrowed by reducing the Rabi frequency $\Omega_{o}$ and leaving all other parameters otherwise fixed.

Besides a sizable degree of dynamic control, this photonic structure enables one to overcome fundamental difficulties arising from fluctuations in the position and size of
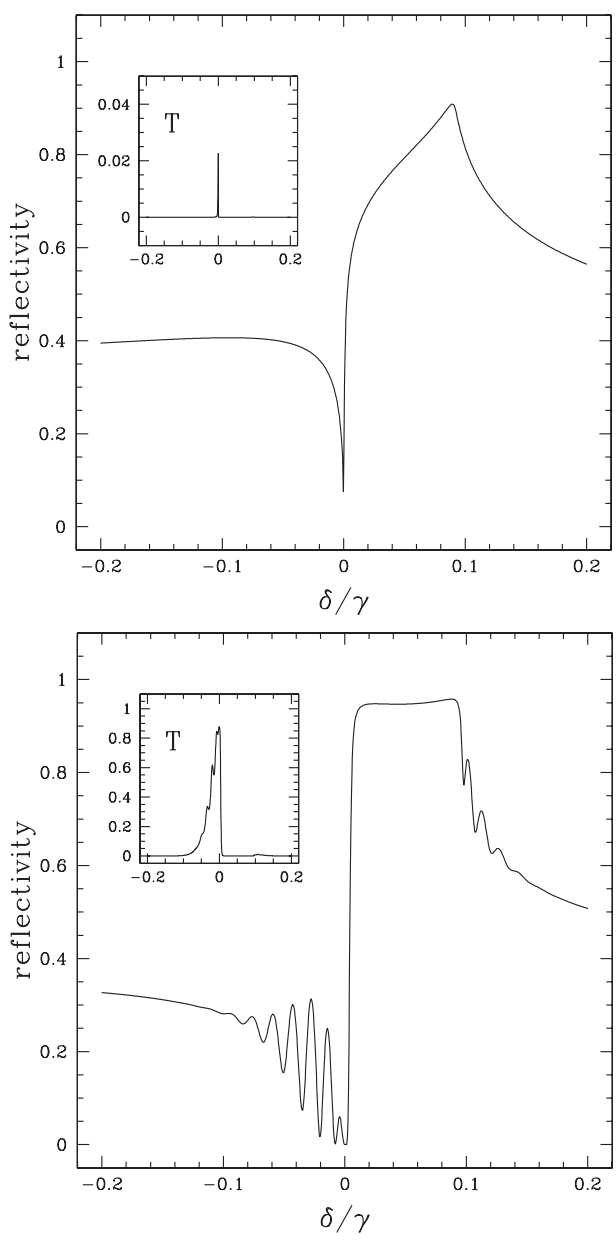

FIG. 2. Induced band-gap reflectivities in a sample of length $L \simeq 2.5 \mathrm{~cm}$. Upper and lower frames correspond, respectively, to the upper and lower gap frequency regions in Fig. 1. The insets show the corresponding transmissivities.

the periodic structure building blocks. In familiar photonic crystals [1] as well as those obtained from atomic density grating, where atoms are confined in optical lattices by dipole forces [27], local disorder generally prevents one from reaching the required fidelity over long ranges [28] altering the Bragg reflectivity and ultimately the gap structure. Conversely, our samples behave much like an ideal atomic Bragg-reflector as the induced optical lattice exhibits an intrinsically perfect periodicity. This, together with negligible dissipation, makes it particularly amenable to the observation of well developed photonic structures [29] which may be used, e.g., to investigate interesting aspects of Bragg processes in cold atomic samples [30]. The accuracy and prompt tunability of these gap structures may instead be employed for quantum light storage [31] and fast optical switching applications [16]. They may, in principle, prove to be useful also as ultralight macroscopic oscillators in demonstrating macroscopic entanglement arising from radiation pressure [32]. At last, extensions from atomic to solid-state materials [33] are highly desir- 


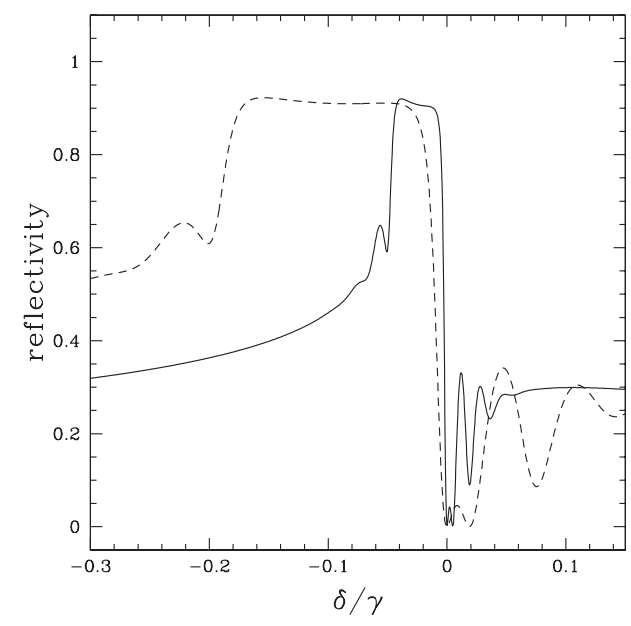

FIG. 3. Tuning of the band-gap reflectivity for different pump intensities with $\Omega_{o}=5 \gamma$ (dashed line) and $\Omega_{o}=2.5 \gamma$ (solid line). The sample has length $L \simeq 6 \mathrm{~mm}$ and the two counterpropagating beams creating the optical lattice are misaligned by an angle $\chi \simeq 20 \mathrm{mrad}$. All other parameters are the same as in Fig. 2 with $\eta=0.05$.

able and, in particular, to doped solids [34] whose level scheme configurations based on impurity levels are shown to work even at room temperature.

We would like to thank F. Bassani, R. Sapienza, D. Wiersma, R. Corbalan, and L. Ditou for fruitful discussions. We acknowledge financial support from the EU (Contract No. HPRICT1999-00111) and M.I.U.R. (Prin 2002-28858 and Accion Integrada Italia-Spagna IT-603).

[1] K. Sakoda, Optical Properties of Photonic Crystals (Springer, Berlin, 2001).

[2] E. Yablonovitch, Phys. Rev. Lett. 58, 2059 (1987).

[3] S. John, Phys. Rev. Lett. 58, 2486 (1987).

[4] K. Bush and S. John, Phys. Rev. Lett. 83, 967 (1999); D. Kang et al., ibid. 86, 4052 (2001).

[5] P. M. Johnson, A.F. Koenderink, and W. L. Vos, Phys. Rev. B 66, 081102 (2002); H. W. Tan et al., Phys. Rev. B 70, 205110 (2004).

[6] S. Harris, Phys. Today 50 No. 7, 36 (1997).

[7] E. Arimondo, Progress in Optics $X X X V$, edited by E. Wolf (Elsevier, New York, 1996), p. 257; A. B. Matsko et al., Adv. At. Mol. Opt. Phys. 46, 191 (2001).

[8] F. Silva et al., Europhys. Lett. 51, 286 (2000).

[9] B. Bailey et al., Nature (London) 366, 44 (1993).

[10] J. Fleisher et al., Nature (London) 422, 147 (2003).

[11] C. Mennerat-Robilliard et al., Phys. Rev. Lett. 82, 851 (1999).

[12] E. Lundh and M. Wallin, Phys. Rev. Lett. 94, 110603 (2005).

[13] L. Pitaeskii and S. Stringari, Bose-Einstein Condensation (Clarendon Press, Oxford, 2003).
[14] A. André and M.D. Lukin, Phys. Rev. Lett. 89, 143602 (2002).

[15] I. Friedler, G. Kurizki, and D. Petrosyan, Phys. Rev. A 71, 023803 (2005).

[16] A. Andre et al., Phys. Rev. Lett. 94, 063902 (2005).

[17] M. Bajcsy et al., Nature (London) 426, 638 (2003).

[18] Absorption blurs in general the distinction between photonic bands and band gaps. See, e.g., A. Tip et al., J. Phys. A 33, 6223 (2000).

[19] See, e.g., C.S. Adams et al., Prog. Quantum Electron. 21, 1 (1997); M. Greiner et al., Nature (London) 415, 39 (2002).

[20] M. Born and E. Wolf, Principles of Optics (Cambridge University Press, Cambridge, 1980), 6th ed.

[21] F. Bassani and G. Pastori Parravicini, Electronic States and Optical Transitions in Solids (Pergamon Press, Oxford, 1975).

[22] B. Batterman et al., Rev. Mod. Phys. 36, 681 (1964).

[23] The case of a perfect standing wave considered here is only partially relevant to the experiment in Ref. [17] where, unlike in the present case, a hot atomic sample $\left(T=90^{\circ} \mathrm{C}\right)$ has been used and where then Doppler effects are important.

[24] In the absence of absorption $R$ is unity within the gap, while when dissipation is present the absorption $A$ is given by $1-R-T$.

[25] A different level configuration with a highly off-resonant standing-light pump wave may also be employed to reduce absorption, yet yielding an even narrower gap [14].

[26] In Fig. 2 we used a slight misalignment $\chi \simeq 5$ mrad. Also note that in Figs. 2 and 3 the resonant wave vector $2 \pi / \lambda_{o}$ is, respectively, slightly smaller and larger than the Brillouin zone boundary $k_{B}=\pi / a$. Here the pump beam wavelength is $\lambda_{c}=780.778 \mathrm{~nm}$.

[27] See, e.g., I. Deutsch et al., Phys. Rev. A 52, 1394 (1995); D. V. van Coevorden et al., Phys. Rev. Lett. 77, 2412 (1996), where, unlike in our case, band gap modulations result from redistribution of the atoms position within the cloud.

[28] A. Koenderink and W. L. Vos, Phys. Rev. Lett. 91, 213902 (2003).

[29] Extensions to either 2D or 3D structures involving a higher number of control beams [19] or to other samples are certainly likely. Other species such as $\mathrm{Na}$ [cf. L. V. Hau et al., Nature (London) 397, 594 (1999)] and Sr [cf. G. Ferrari et al., Phys. Rev. Lett. 91, 243002 (2003)] may be used in place of $\mathrm{Rb}$.

[30] S. Slama et al., Phys. Rev. Lett. 94, 193901 (2005).

[31] M. Lukin, Rev. Mod. Phys. 75, 457 (2003).

[32] See, e.g., S. Mancini et al., Phys. Rev. Lett. 88, 120401 (2002).

[33] G. Serapiglia et al., Phys. Rev. Lett. 84, 1019 (2000); M. Artoni et al., Europhys. Lett. 49, 445 (2000); E. Kuznetsova et al., Phys. Rev. A 66, 063802 (2002); S. Chesi et al., Phys. Rev. Lett. 91, 057402 (2003).

[34] A. V. Turukhin et al., Phys. Rev. Lett. 88, 023602 (2002); M.S. Bigelow, N. N. Lepeshkin, and R. W. Boyd, Phys. Rev. Lett. 90, 113903 (2003). 